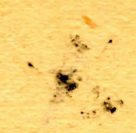

ILLUSIONS OF VISION AND THE CANALS OF MARS

By Professor ANDREW ELLICOTT DOUGLASS

[Reprinted from The Popular Science Monthly, Vol. LXX, May, 1907] 
[Reprinted from The Popular Science Monthly, May, 1907.]

\section{ILLUSIONS OF VISION AND THE CANALS OF MARS}

By PROFESSOR ANDREW ELLICOTT DOUGLiSS

UNIVERSITY OF ARIZON

'LHAT fascinating mystery, the planet Mars, will again approach the earth this summer. Again the nightly watcher will note the diminishing snow caps at the poles, the dark areas of vegetation, enlarging with the welcome moisture, and, perchance a cloud or two that, lingering over the cold Martian night, is dissipated in the sunrise heat, revealing thus its character.

Again also will hundreds of fine dark lines appear, which from their straightness and artificial appearance, seem to attest the existence of highly intelligent beings upon our neighbor.

It is right and natural that we should first regard these faintest of markings as realities upon the planet. The writer can certify to their apparent genuineness, for he has pictured numbers of them in half a dozen favorable oppositions since 1892. To him they were real until time proved that in the faintest markings astronomers failed of satisfactory agreement. In the larger markings, and even in the larger canals, conflicts of evidence do occur, but are never troublesome. One may confidently say that such realities do exist. But with the very faint canals whose numbers reach occasionally well into the hundreds, discordance reigns supreme, and it is frequently found that different drawings by the same artist antagonize each other across the page.

Considerations along these lines led the writer to study seriously the origin of these inconsistent faint canals by the methods of experimental psychology, and the application of those methods has resulted in a new optical illusion and new adaptations of old and well-known phenomena, all of which apply profoundly to the case in hand. Their description and application follow.

\section{HaLO}

The most important of these phenomena is the halo.

To observe this, place Fig. 1 at a distance of six to eight feet from the eye and look at it from time to time, taking care to avoid fatigue. Around it will appear a whitish area limited externally by a faint dark line forming a perfect circle, as if traced by a pair of compasses. This external ring or secondary image has a sensible width and appears blackest on its sharp inner edge. When once caught, which is usually 
at the first view, it is a striking phenomenon. I find on the whole that trained eyes are the ones which see it most quickly.

A more beautiful and elegant way of making the experiment is by standing a black-headed hat pin in the middle of a white-walled room, and looking at it against the distant white background. Around the head of the pin will then appear this halo, more beautiful than before, suspended in mid-air, in the good old-fashioned manner of saintly halos.

The experiment described above gives the 'negative' halo. It will be generally referred to in this article, because it is more easily seen than the 'positive.' The 'positive' form of the halo, however, is most readily seen by a similar method. Let a whiteheaded pin be substituted for the other, and looked at against a black background. Similarly, a

Fig 1. This Spot SHOULD BE VIEWED From a Distance of Six or Eight FeEt, with care to avoid fatigue or after-images, in oıder to see the fine dark halo ring about it at the distance indicated by the smaller dot.

white circle is seen. The difficulties in this case arise from the reflections on the head of the pin and its generally less even illumination.

The effect, however, is the same. Extending all round the head of the pin at a distance of about $7^{\prime}$ of arc (one inch at a distance of 500 inches) is an intensified zone in which the color of the background appears stronger; and outside of that a reduction zone, or ring, or secondary image, in which the intensity of the background is reduced by the addition of some of the color of the spot observed.

In order to find the cause of this halo, many tests were made, of which the first was upon the size of the central spot. It was found that the distance from the edge of the spot to the secondary image is constant; that the width of the secondary image increases to some extent with the size of the spot, and that the intensified area increases its intensification with the size of the spot. If the spot is so small as to be barely visible, the halo may still be seen, but the intensified zone then appears of the same intensity as the background.

If the spot is enlarged sufficiently, both positive and negative halos are seen along its margin, one outside and one inside, so that in a straight line separating light and dark areas, the positive halo may be seen in the dark area, and the negative halo in the light. If two small spots are placed so that their halos intersect, the halo of each voL. $\mathrm{Lxx} .-30$ 
may usually be seen complete. If the spots are larger, the halos can not be traced within each other's precincts, and on enlarging the spots still more, they soon act as one mark with regard to the halo, which assumes an elliptical form around them. From these and other experiments along the same line, it appears that the intensified zone or white area, as I shall generally call it, referring to the negative experiment, displays an increased sensitiveness to presence or absence of color of the spot looked at, but a decided deadening in the perception of details.

My first idea in regard to this halo was that it came to life like the camera ghost, from reflections between lens surfaces in the eye, but $\dot{\mathrm{I}}$ found that it could be produced through any portion of the crystalline lens. A pin hole $1 / 50$ inch in diameter passed before the pupil of the eye demonstrated this.

It then seemed possible that some form of halation in the membranes close to the retina might produce this effect. The common

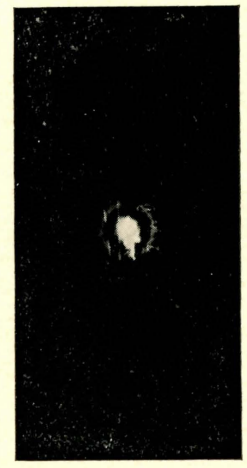

Fig. 2. Photographic Halation Ring a Bout CandLe Flame, formed by reflection inside the glass plate on which the picture was taken, very similar in its appearance to the halo here described. photographic halation ring, which closely resembles it, is produced by reflection from the back of a glass plate but can only occur under certain conditions. This halo, however, occurs on all margins and can not be due to that cause.

At this stage, a certain ' chromatic ring,' described below under that heading, was observed, and suggested some obscure color conditions as the cause. Hence, color tests were made in large numbers, and the black spot was tried on different colored backgrounds without effect. Different colored spots against a dark background were also observed without effect, save that the secondary image when sufficiently bright was seen to be of the color of the spot itself; therefore color was not responsible for the halo.

But these color observations opened up a very interesting line of study. The color tests had to be made in the positive form with all the attendant difficulties of fatigue and after-images. It was found that a short gaze at a red disk on a black background, followed by a slight movement of the eye to one side, carried away a dark green after-image of the disk surrounded by a red margin, about the size of the intensified zone. This intensified zone became still more con- 
spicuous by longer fixation of the gaze upon the colored spot. To observe this, half-inch disks of red, yellow, green and blue paper were pasted vertically on ends of long needles and placed in strong lamplight at a distance of eight feet from the eye. After long unwinking gaze at one of these disks, until general color sensitiveness seemed to be disappearing and the color of the disk itself seemed to be spreading out around it, a quick closing of the eye, or the mere placing of a sheet of paper close before the open eyes, revealed a very interesting succession of changes, as follows:

1. A black or dark green disk with a limited red margin filling the intensified zone, limited by the dark halo. This effect lasted for a very brief instant of time, like the common positive after-image.

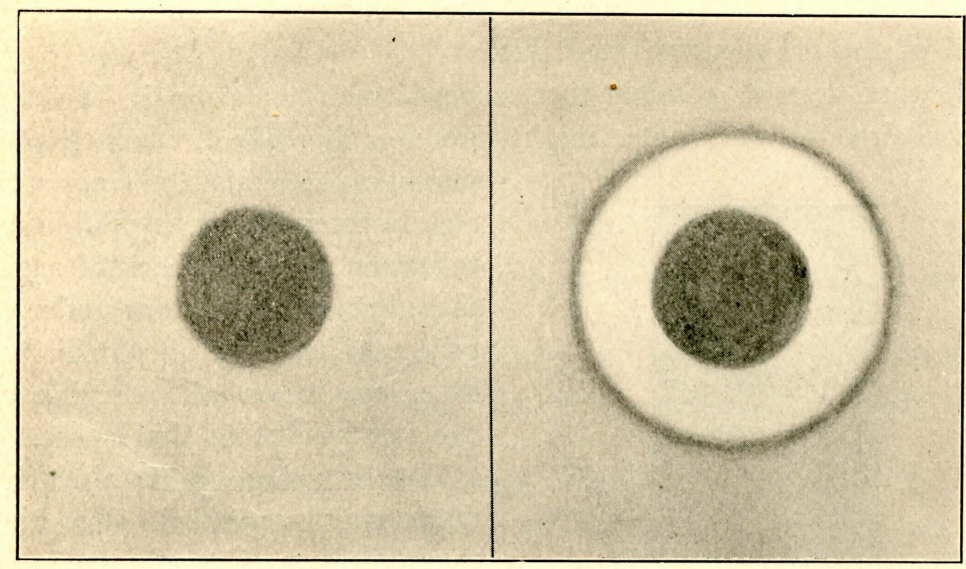

Fig. 3. 'Dot' Mote outsine the YELLOW SPOT.

Fig. 4. 'Dot' Mote in Yellow Spot BUT NOT IN FOVEA.

2. The outline soon reappeared; the red disk and all white objects taking a dark indigo-blue color, the remainder of the field being a bright yellow. This effect might last a minute or two.

3. During the height of this effect a negative halo appeared for a time around the dark after-image of the disk at the usual distance of $7^{\prime}$. The success of this experiment depends largely upon steadiness of vision and avoidance of winking. The determination of the effect of different colors and conditions offers a fine field for investigation.

The next test with a view to locating the cause of this halo phenomenon was made on motes that so often float by the line of vision. This was done by looking at a highly-illuminated area through a small pin hole held close to the eye. Three classes of motes were observed: First, the usual cell fragments and groups; second, rapidly moving objects probably of similar character, and, thirdly, minute black dots which from their motions seemed to be located in the same region as 
the first, probably not far in front of the retina. On this last class, some beautiful halo phenomena were observed.

When one of these spots was outside a region identified as approximately the yellow spot, it appeared as a circular dark area of some $30^{\prime}$ diameter as shown in Fig. 3. When it came within the yellow spot, it became lighter, and was surrounded by the halo, with its intensified zone and secondary image well defined as in Fig. 4. When, however, it came within the region of most distinct vision, which was very rare, it gave the most beautiful halo effect I have yet seen. It had a dense, black spot in its very center, usually well rayed; then, a light zone limited by an intense black ring, which in turn produced its own complete halo. This form is shown in Fig. 5.

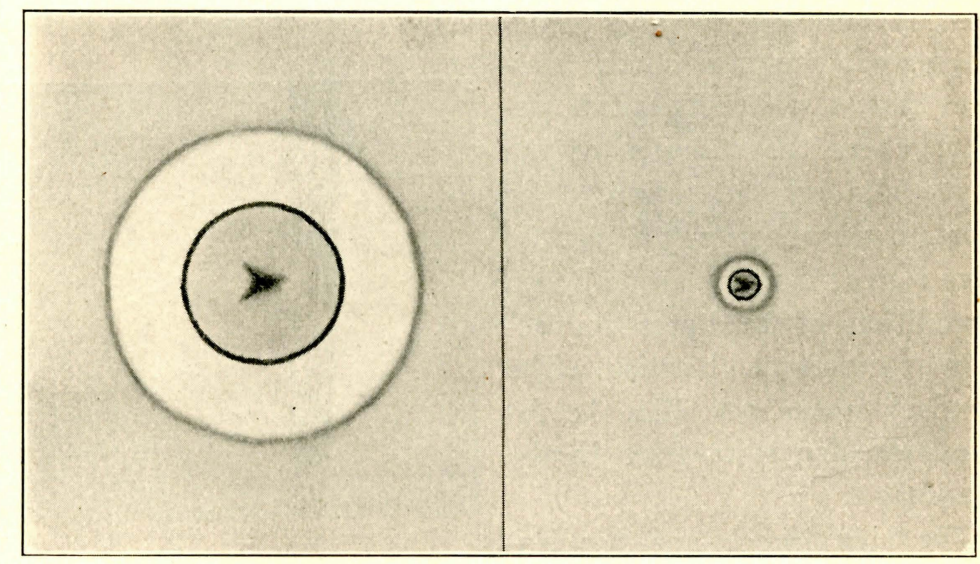

Fig. 5. 'Dot' Mo'te in Fovea.

Fig. 6. Same as Figures 5, viewed at Close Range. Notice different length of rays compared to diameter of ring.

This mote observation is by no means easy. I have often waited fifteen minutes for a mote of this type to appear, and only once have I. kept one in sight for any length of time. It then remained in the center of vision for at least twenty minutes. Usually, they float past the center of the vision and give one only a brief view. The size of pin hole used is $1 / 50$ inch. With a much larger hole, say $1 / 20$ inch, they become blurred. By getting near a large lamp shade so that a wide angle of light is viewed, they are best discovered. Then one may retreat from the light and view them as illustrated in Figs. 3, 4 and 5 .

The rays observed in the central spot are very interesting. Their length offers a means of measuring the height of the spot above the retina. A short calculation upon approximate data results in 0.002 inch as the distance of the spot from the retina.

It is true that these mote observations require great patience, but 
the beauty of the phenomena repays the effort. There is a sharpness and a density about the inner halo around the spot itself which does not characterize the ordinary outer halo. For such differences I have no explanation to offer.

Not only is the cause of these details very difficult of detection, but the origin of the whole halo phenomenon is equally so. It probably lies in the obscure reactions that change light waves into nerve impulses. One thing which the intensified zone does do is to help correct for rays which the irregular refraction of the eye scatters across a margin; and so this light area fulfils some psychological necessity.

The fact that in the first flash of after-images this zone becomes occupied by the color of the object looked at (like the common positive after-image) suggests that it is a zone in a condition of expectant attention with reference to that color. If, for example, a red disk is observed, the nerves that perceive that color are in full activity, where the stimulus of the image falls on the retina. For a certain distance away from the active retina, they are aroused into a condition of readiness for activity or expectant attention. The secondary image acts like the fatigue area, for it reverses in the atter-image.

The significance and application of the phenomena are easier.

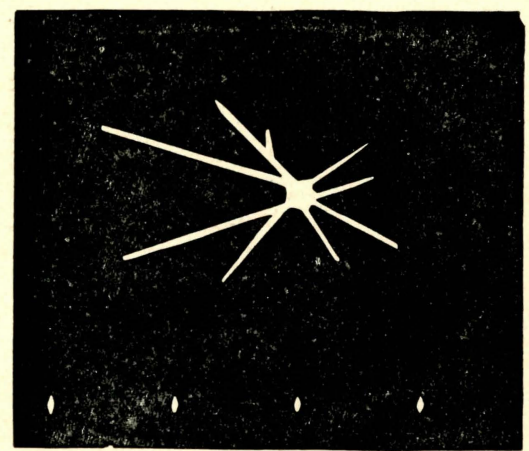

Fig. 7. Stei.lar Rays.

From the psychological standpoint, its immediate application is to questions of contrast. Contrasts are divided into two classes: First, successive contrast, due to fatigue and rest; second, simultaneous or marginal contrast, now seen to be a subordinate part of this halo phenomena. Marginal contrast has been long known, and its afterimage, the 'Lichthof' of Hering, has been described. The fact that the halo phenomenon definitely limits the region of marginal contrast and displays a secondary image in a definite position proves it to be the more fundamental phenomenon. We have here, therefore, a new illusion of interest to psychologists and of great significance in its application to astronomical work.

\section{RAYS}

Unlike the halo, the ray phenomena are familiar and involve no new principle, but the idea of rays around a black spot is new to me, and quite as important as the halo in its application to visual work by telescope or microscope. As all know, the rays on a star are pro- 
duced by irregular refraction in the eye, originating in what are known as the stellate figures. The figures seem to be construction lines, as it were, in the crystalline lens, and develop during its growth. They are permanent in form, when adult years are reached, and may be

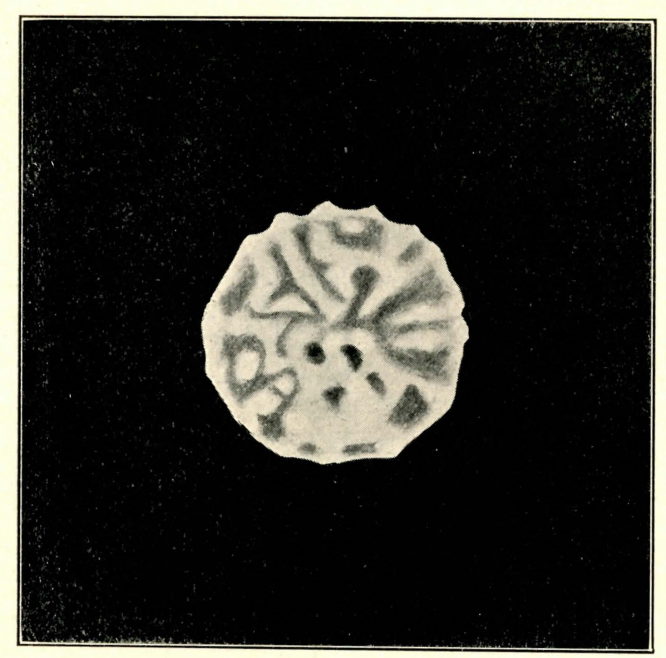

Fig. 8. Siruetere Lines in Crystalline Lens.

seen with ease by the methods commonly explained in books upon experimental psychology.

If white rays may be seen around a white star on a dark background, then black rays must be visible around a black spot on a white background. They may be easily seen by screening the greater part of the pupil and allowing light from a black spot to pass through

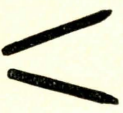

FIG. 9. RAYS ON A BLACK SPOT OBTAINED BY SCREENING ALL THE PUPIL EXCEPT THE MARGIN OF THE (LEFT) SIDE. These rays are the two long rays on the left in Fig. 7. its margin. This is best done by a small circular screen on the point of a needle. By slight perseverance all the principal rays seen on a star may be perceived on the black spot. These are always present in the eye, but are not commonly perceived, because they are drowned out in the lighter background, and habit compels us to disregard them. Their importance in astronomical work is at once evident when I state that with the head in a definite position, I found it easier to see certain lines on the planet Mars and those easier lines coincided in direction with the two black rays represented in Fig. 9. It is evident that observations made with the greatest possible care ought to show these canals like marks, and if two of these rays be parallel, as may easily happen in an astigmatic eye, some of the canals should appear double. 
Irradiation.-Next to the black rays in importance is the matter of irradiation as analyzed by means of ray forms. The method of investigation is as follows: Make a small hole in a window blind and observe the sky through it by different distances. From near-by the outline of the hole is well perceived, but, as one draws away, the rays soon obscure the hole itself, so that its form and size can not be perceived. At these different distances, the width of the rays varies with the true angular size of the hole. For example, I found at ten feet an irradiation of $2^{\prime}$ and at three feet an irradiation of $6^{\prime}$, because at the nearer point the rays are three times as wide and overlap each other at three times the distance from the hole. Irradiation then is the merging together of the rays, and on any straight line of separation, is proportional to the total ray light on the corresponding hemisphere about a star.

Color, size, intensity and perfection of eye are positive factors in irradiation. The negative factor is the background, and the result depends upon the sensitiveness of the eye at the time of observation. Some general results we can see at once. Irradiation is not necessarily the same in any two eyes or in any two directions. It varies with fatigue of the retina and probably with use of the eye in some unusual position, producing unusual conditions of pressure upon the eye-ball. Ordinarily, its amount depends directly on contrast between the areas observed and on the size of the central nucleus of rays in the desired direction. This nucleus must not be assumed to be centrally located on its source.

Best Part of Lens.-A very important bit of information derived in this study of the rays is the location and size of the best part of the crystalline lens. This is done by trying smaller and smaller diaphragms over the eye until the rays cease to appear. They will be found to persist in rudimentary form even when the diaphragm is as small as 1/16 of an inch. This is of great significance in telescopic and microscopic work, because it shows how small the emergent pencil of light must be to avoid the excessive formation of rays. Even at best, they can not be hindered entirely. The use of lower powers with large emergent pencil is therefore very dangerous. The optically superior part of the lens occupies a small irregular area near the center with irregular extensions out toward the margin. Even the best part is far from perfect.

Detached Spots.-An interesting variation of stellar rays has been observed at least in one case. A gentleman drew for me the rays as they appeared to his eye in the experiment described above (see Fig. $\mathrm{r}$ ), and while working asked me if I had placed a number of smaller pin holes around the large one. Fig. 10 represents this. It is perfectly possible for detached spots of this kind to be produced by some 
irregularity of the lens structure and thus to supply illusive satellites to planets or fictitious companions to double stars.

\section{Chromatic Rings}

The illusive chromatic rings which follow do not bear so much on questions of Martian topography as the preceding halo and rays. Yet they are interesting of themselves and have an influence on color estimations. The first is the broad prismatic ring which extends from

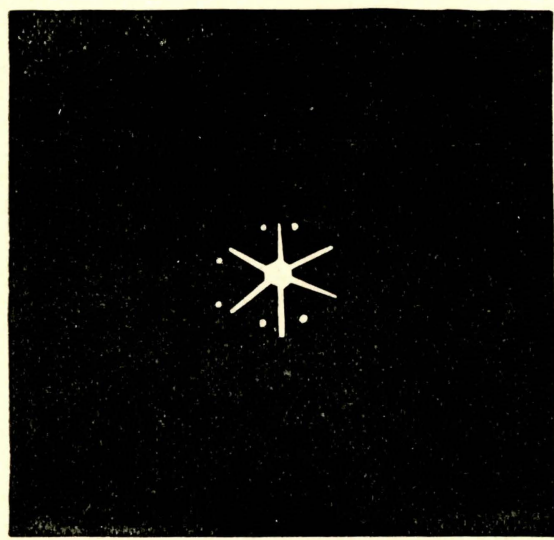

Fig. 10. Rays and Detached SPOTS. about $312^{\circ}$ to $5^{\circ}$ from the source of light with red on the outside and green or blue on the inside. This shows well on any brilliant light such as the full moon or a bright electric light.

The second is a narrow blue ring, of interest on account of its beauty. It is best seen on an electric arc light of intense blue color-and the less continuous spectrum the light shows, the better. Standing at a distance of one hundred and fifty or two hundred feet, one may see a beautiful narrow blue line forming a circle fully two feet in diameter about the light. As the color of the light changes to yellow, which it frequently does, the ring rapidly disappears into the center of the light.

This ring may be seen in the laboratory by passing the blue light of the spectrum through a pin hole. In mid-blue its radius is about $12^{\prime}$. Various experiments show that this illusion is produced at the margin of the pupil by the bending of the blue rays too sharply toward the optical axis of the eye. These rays therefore focus in front of the retina and on reaching it form a blue ring outside of the true image.

\section{Radiating Lines from Near the Center of a Blank Disk}

The only remaining illusion to which I call attention is one of much importance in planetary work, but one for which I shall not attempt an explanation. Frequently in observing a blank white disk, lines have appeared to me to radiate from some point near the center. When first I observed lines of that character, not knowing whether they were really there or not, I considered them genuine and for a long time represented them in the form of a star with four or eight 
rays. At last when they did not show agreement among themselves I concluded they must be illusions. This was verified by specific trial, proving that such lines appear on perfectly blank areas. The rays so observed are sometimes double.

\section{Application of these Phenomena}

Against the obstacles of bad atmosphere, minuteness of detail and faintness, the observer has to wage a hard fight, and it is a matter of congratulation that he sees such faint canal-like marks on the very limit of vision. With full records the public may then discuss the interpretation.

The ray illusion is to me a very satisfactory explanation of many faint canals radiating from those small spots on Mars, called 'lakes' or 'oases.' The only objective reality in such cases is the spot from which they start. The reader will notice that rays on opposite sides of a star are usually in line. So when two lakes or oases lie along such a line they will appear connected by a canal. Nor do the oases need to be very close together. A ray $16^{\prime}$ long to the naked eye appears $4^{\prime \prime}$ long on a planet magnified 240 diameters. With the planet Mars $16^{\prime \prime}$ in diameter the ray then extends one fourth across it. It appears like a canal over one thousand miles long.

I believe the industrious observer has found and will find it difficult to avoid instinctively placing his head in a position favorable to producing combinations of this kind. After he has laboriously memorized the leading details, so that he may recognize what he sees, when, for an instant, Heaven vouchsafes him a brief view, he naturally has a powerful inclination always to observe in the same posture, for he finds that with a slight movement of his head his structure of fainter canals is liable to disorganization. This insistence upon the same attitude is at once understood when we consider a larger part of the faint canals to be due to rays in the eye.

We have here the medicine to prevent this disease in the future. Let the observer constantly vary the position of the head. As soon as the seeing becomes sufficiently good to reveal fine detail, let the movement of the head begin. A rotation through an arc of twenty or thirty degrees ought to be large enough to test thoroughly any fancied combination of canals. Drawings carefully made in this way will have one source of error eliminated.

The halo with its light area and secondary image accounts for details which have no objective reality, such as bright limbs of definite width, canals paralleling the limb or dark areas, numerous light margins along dark areas and light areas in the midst of dark-abundantly exemplified in Schiaparelli's map of 1881-2.

When a ribbon-like mark has sufficient width, it must appear 
double; for the positive secondary image of the adjacent light areas will appear within it. To this end its apparent width to the naked eye must be some $8^{\prime}$ or $10^{\prime}$ (if eyes are alike in this dimension). In a telescope magnifying, say, 400 diameters, this width need be only a little over $1^{\prime \prime}$. If the planet is $16^{\prime \prime}$ in diameter (a rough average of its favorable position in recent years) this will amount to closely $10^{\circ}$ on its surface. Now the double canals of Schiaparelli, in 1881-2, and of Perrotin and Thallon, in 1886, are frankly of this width and, I believe, are due to this cause. In any case the test to be applied is evidently the relation between the apparent width of a double and the radius of the halo illusion. The prevention of error in the future will evidently be the application of different powers to each canal, particularly a low power which will make its width appear less than $6^{\prime}$. This must be done with care for low powers increase the number of rays.

The halo illusion is also responsible for marginal canals. When a dark area becomes $6^{\prime}$ or $8^{\prime}$ wide, it appears double, having a light interior and dark edges. With any increase of width the dark edges, giving the effect of the marginal canals, remain. Hence along the edge of any dark area there appears a fictitious canal. Professor E. W. Maunder observed this in his excellent artificial planet study of a few years ago. I believe that high powers by reducing contrast will help to eliminate this error.

The mention of the chromatic rings draws attention to chromatic aberration in the eye and in the telescope. This effect in the telescope is so great that colors in a refracting telescope are not in the least trustworthy. The blue-green tint attributed to the dark areas on Mars is a product of the telescope. Its existence on our neighbor can only be verified by the use of a reflector.

Thus in conclusion we see that there are fundamental defects in the human eye producing faint canal illusions, that these have worked serious injury to our observations in the past and that in the future they may be avoided chiefly by the simple expedients of varying the position of the head and using a wide range of magnifying power. 